\title{
SUPERCRITICAL FLUID THERMODYNAMICS FOR COAL PROCESSING
}

PROJECT NO. DEFG22-88PC88922

PRINCIPAL INVESTIGATOR: Prof. Frank van Swol University of Illinois at Urbana-Champaign Department of Chemical Engineering

SUBCONTRACTOR: Prof. Charles A. Eckert Georgia Institute of Technology School of Chemical Engineering (404) 853 - 9344

INITIATION DATE: September 15, 1988

Period Covered by Report: July 1, 1990 - September 30, 1990

\begin{abstract}
Because of their unusual solvating and mass transfer properties, supercritical fluids show potential for a variety of coal processing applications. We have established a database of coal model compound equilibria; to add to this database, we have developed and are testing techniques of measuring entrainer effects on solubility rapidly. In addition, we have used fluorescence spectroscopy to study the nature of entrainer effects on a molecular level. The solubility and spectroscopic measurements are being used in the development of an equation of state that includes both physical and chemical interactions; we are currently testing the equation. The equation of state will be used to predict solubility behavior so systems can be designed for the processing of coal with supercritical fluids.
\end{abstract}

\section{DISCLAIMER}

Wh account of work sponsored by an agency of the United States This report was prepared as an account of work sponsored by an agency of thereof, nor any of their Government. Neither the Unired States Government nor any agency thercol liability or responsiemployees, inakes any warranty, express or implied, or assumes any legal liability or roduct, or bility for the accuracy, completeness, or usefulness of any information, apparalus, product, process disclosed, or represents that its use would not infringe privalely owned right trademark, ence herein to any specific commercial product, process, or service by trade natorsement, recommanufacturer, or otherwise does not necessarily constitue or any agency thereof. The views mendation, or favoring by the United States Government or any agency therect those of the and opinions of authors expressed herein do not 


\section{OBJECTIVES}

The main objective of this research is to develop an equation of state that can be used to predict solubilities and tailor supercritical fluid solvents for the extraction and processing of coal. To meet this objective we have implemented a two sided approach. First, the solubility of coal model compounds in higher temperature fluids, polar fluids, and fluid mixtures systems must be determined. Second, the intermolecular interactions between the solute and the SCF solvent must be investigated (using spectroscopic techniques) in order to understand them better and develop an EOS that accurately reflects the true molecular makeup of the solution.

Supercritical fluids (SCF) show potential for coal processing because they possess some unique properties. A SCF is quite dense, which gives a large capacity for solutes; it has high diffusivity and low viscosity, which makes it an ideal medium for efficient mass transfer; and the fluid is highly compressible, which yields large solubility changes with small changes in temperature and pressure and suggests extraordinary selectivity possibilities.

Many of the SCF investigations to date have been with relatively mild, low-temperature fluids like carbon dioxide. However, any process for coal conversion will require more aggressive, higher temperature and more polar SCF solvents like ammonia, methanol, and butane. In addition, we have found that the addition of a small percentage of a different component that specifically interacts with the solute (an "entrainer") can dramatically increase the solubility of heavy organic solutes. Therefore, we are proceeding with the measurement of solubilities in these novel fluids and fluid mixtures.

Liquefaction and processing of coal liquids will involve a situation where one (or more) of the phases present is liquid, and this situation poses special problems in both characterization and measurement. To investigate these important systems we have developed a packed column chromatographic technique to determine distribution coefficients of solutes between the liquid and SCF phase.

Finally, in order to develop an equation of state that can be used to predict as well as correlate solubility data, an understanding of the actual intermolecular interactions is required. To probe those interactions we have developed a high pressure fluorescence spectroscopy apparatus to probe those interactions. From the spectra information can be derived about the strength of both solute/solvent and solute/solute forces and how they change with proximity to the critical point. Both the solubility and spectroscopy data are vital to the development of an equation of state model that will take into account both the physical and chemical forces that are important in SCF solutions. 


\section{PROCIRESS THIS QUARTER}

\section{PHASE BEHAVIOR}

In past quarters, we compiled an extensive database of coal model compound solubilities. Although entrainer modified SCF's show much promise in facilitating difficult separations, relatively little data exist on the effects of these modifiers. Therefore, we are now extending this database by using two novel techniques for measuring solubilities in supercritical fluid solutions containing cosolvents.

We are testing a chromatographic technique to rapidly measure entrainer effects. Currently, we are calibrating the instrument by making measurements of retention times (which are directly related to solubility) of well characterized compounds in pure $\mathrm{CO}_{2}$. Cosolvents will similarly effect retention time, giving us a direct measure of the cosolvent effect.

We are also constructing a new apparatus that will measure solubilities using UV absorbance. Identical high pressure cells will be filled with a known concentration of a solute and the absorbance measured at some temperature and pressure, giving the composition dependence of the extinction coefficient. The absorbance will also be measured in another cell having an excess of solute, thus yielding the solubility of the solute.

These measurements along with fluorescent spectroscopic studies of the same systems will yield much insight on the nature of interactions on the molecular level and their macroscopic effect. This knowledge coupled with an accurate chemical physical model will allow inexpensive and rapid design and development of coal treatment processes.exploiting this database to refine and test a new equation of state that we have developed. This equation can be used in the design of coal treatment processes. Chemical forces can play an important role in phase equilibria, particularly in systems with entrainers (such as methanol) where hydrogen bonding occurs, yet the current commonly used equations of state consider only physical forces between molecules. Our equation of state includes both chemical and physical terms.

Tw'o distinct opportunities exist in the area of association models of supercritical fluid solutions. First, experimentally determined equilibrium constants are needed to reduce the number of adjustable parameters in the association model and provide a better test of the ability of the model to predict SCF phase behavior. Spectroscopic measurements will help us not only to determine the equilibrivin constants, but will better inform us of the reactions that must be considered in the model. Second, association models may be an appropriate way to describe the clustering of solvent molecules around a solute in the region near the critical point that is highly compressible. We are currently working on both of these opportunities. 


\section{INTERMOLECULAR INTERACTIONS}

The steady state fluorescence measurements showing exciplex formation in the system Naphthalene/Triethylamine(T-A)/CO $\mathrm{CO}_{2}$ indicate a substantial pressure effect on the rate constant for exciplex formation in the near critical region. Using a proposed mechanism for the fluorescence of naphthalene in the presence of triethylamine (Van and Hammond, 1978), the ratio of quantum yields in the presence and absence of TEA can be related to the rate constants and concentration of triethylamine. The quantum yield can be estimated by the area under the fluorescence peak and in Figure 1 the ratio of normal fluorescence area of naphthalene without and with triethylamine is shown as a function of TEA concentration at $35^{\circ} \mathrm{C}$.

In the thermodynamic framework of transition state theory the rate of a bimolecular reaction will be a function of pressure, given by

$$
\frac{\partial \ln k}{\partial P}-\Delta v^{*} / R T
$$

where $\Delta v^{7}$ is the activation volume, given by the difference in partial molar volumes of the transition state and the substrates. In the mechanism, the only bimolecular reaction is the formation of the exciplex from an excited naphthalene molecule and a TEA molecule with rate constant $\mathrm{k}_{3}$ and the difference in partial molar volumes may be significant so we will concentrate our efforts on this reaction. Unfortunately, the data do not give the pressure dependence of $k_{3}$ but rather that of a complicated ratio of rate constants. However, with the assumption that only $k_{3}$ depends on pressure, the natural logarithm of the slopes in Figure 1 is just the logarithm of $k_{3}$ plus a constant and therefore $\partial(\ln ($ slopes from Figure 1$)) / \partial P$ should be proportional to $-\Delta \mathrm{v}^{*}$. The quantity $\partial\left(\ln \mathrm{k}_{3}+\right.$ Constant $) / \partial \mathrm{P}$ was determined numerically from the values in Figure 1 and is plotted as a function of pressure in Figure 2 for $35^{\circ} \mathrm{C}$. Notice the sharp peak which is in the highly compressible region nearer the critical point.

The activation volume can be estimated with an equation of state by' calculating the partial molar volumes of substrates and complex. We used the Peng-Robinscin equation to estimate the equation of state contribution to the partial molar volumes and also included a term due to electrostriction of the solvent about the substrates and complex. Calculated activation volumes are shown in Figure 3 for $35^{\circ} \mathrm{C}$ and $50^{\circ} \mathrm{C}$. The contribution of the electrostriction term is overwhelming and causes the activation volumes to be large and negative, especially in the compressible region at $35^{\circ} \mathrm{C}$. Since $\partial \ln \mathrm{k}_{3} / \partial \mathrm{P}$ is proportional to the negative of the activation volume, the calculations predict a large positive peak in $\partial \ln \mathrm{k}_{3} / \partial \mathrm{P}$ in the region of highest compressibility which is in remarkable agreement with the experimental data. This analysis suggests that taking the pressure effect on the reaction rate is not only appropriate but also necessary when looking at reactions in highly compressible SCF's. 


\section{PLANS FOR NEXT QUARTER}

We will measure cosolvent effects using our new supercritical fluid chromatographic technique. The nature of this device should allow us to procure many data rapidly. In addition, we shall continue construction of the UV absorption apparatus for measuring solubilities. Also, we will continue testing and improving our new chemical-ph, sical equation of state based upon our solubility and spectroscopic data.

Many examples exist of solute-solute interactions in SCF solutions even when both solutes are fairly dilute $(<1 \%)$. We propose to study the formation of excimers (excited state dimers) of pyrene in SCF solutions to explore the nature of solute-solute interactions in very dilute systems $\left(10^{-6}\right.$ mole fraction). This information should provide a unique complement to the data on density enhancements presented earlier.

\section{SUMIAARY}

Supercritical fluids (SCF) show potential for a variety of coal processing applications. To determine the phase behavior to be anticipated in these systems we have assembled a database of solubilities of representative coal compounds in supercritical fluids. To add to this database, we have developed and are testing techniques to measure the effects of entrainers in SCF solutions rapidly. In addition, we have used fluorescence spectroscopy to determine the nature of the intermolecular interactions, such as solute-entrainer interactions, in systems of simple aromatics in SCF carbon dioxide.

Future work will include further investigations of entrainer effects using supercritical fluid chromatography, fluorescence spectroscopy, and UV absorption. This information and the database of solubility measurements will be used in the further development of an equation of state model to predict solubilities for the design of coal treatment processes. 


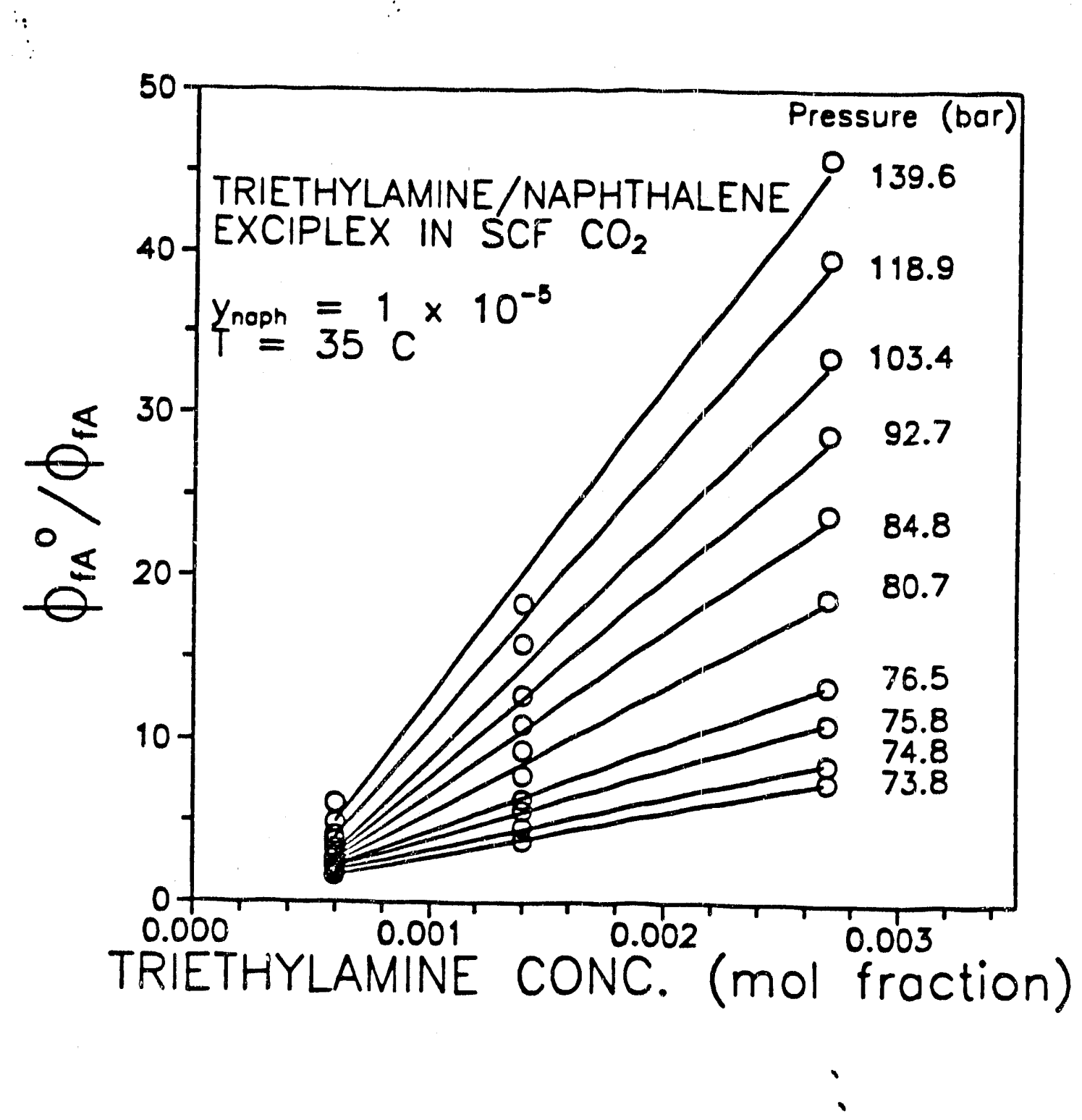

Figure 1 Ratio of normal fluorescence area of naphthalene without and with triethylamine in $\mathrm{SCF} \mathrm{CO}$ at $35^{\circ} \mathrm{C}$ 


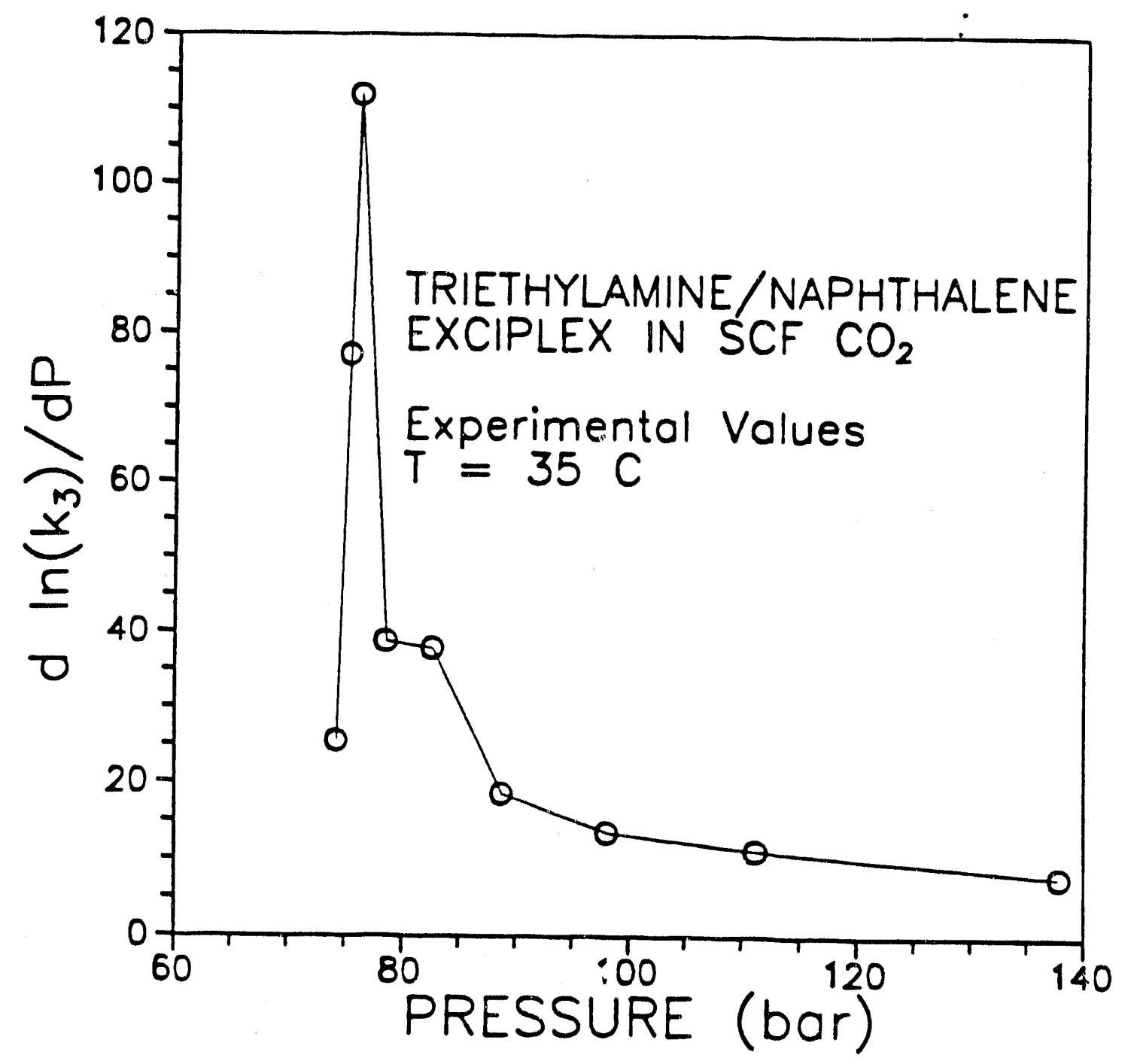

Figure 2 Pressure dependence of the rate constant for exciplex formation numerically determined form the slopes in Figure 1. The line connects experimental points. 


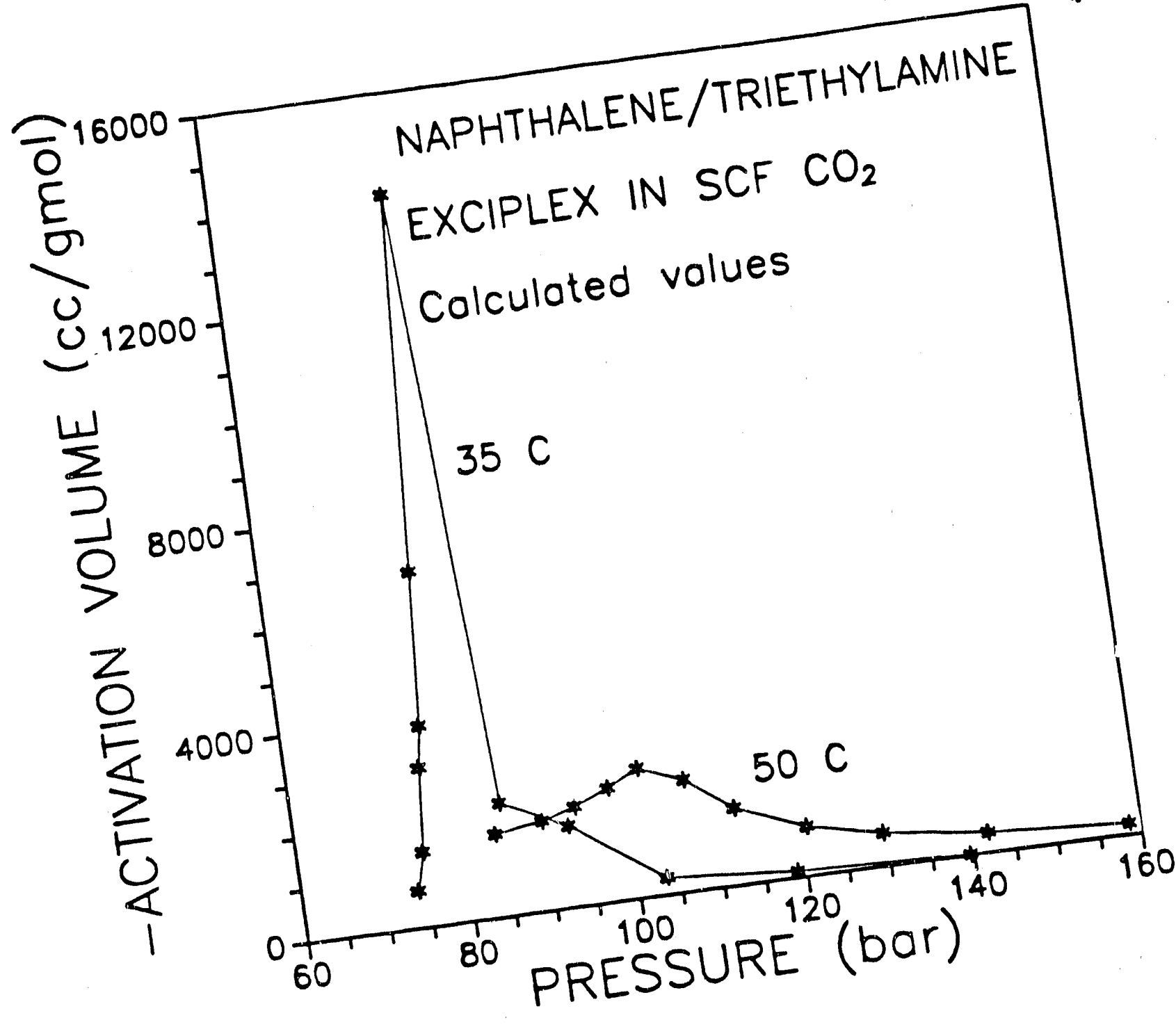

Figure 3 Negative of the activation volume calculated from the sum of the con
from the Peng-Robinson equation of state. The line connects calcula 

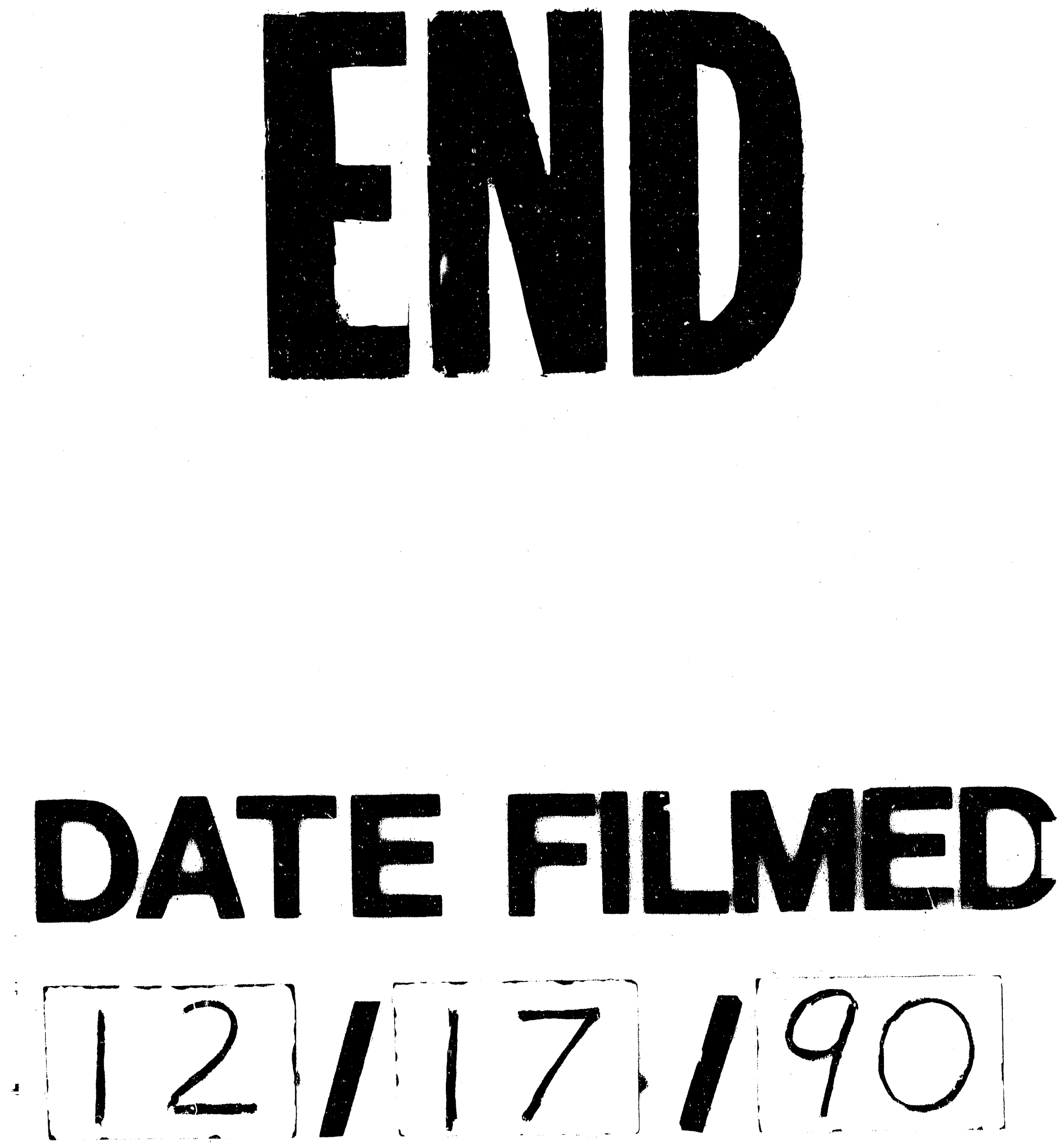
1 